\title{
Etat Environnemental Des Sols De La Region De Casablanca
}

\section{I.Benazzouz,}

\section{Talbi,}

Laboratory of Analytical chemistry and physical chemistry of materials Faculty of Sciences : Ben m'sick University Hassan II, Casablanca, Morocco

\section{N. Saber,}

High School of Technology Sidi Bennour, Morocco

Laboratory of devlopement and research

Doi:10.19044/esj.2020.v16n27p90 URL:http://dx.doi.org/10.19044/esj.2020.v16n27p90

Resumé

La pollution chimique, dans la région de Casablanca impacte la qualité des sols agricoles. Cinq sites de la région de Casablanca sont étudiés : les sites d'Oued Hassar, Oued Merzeg, Bouskoura, Benslimane-Mohammedia et Bouznika. L'objectif est l'analyse comparative de cinq éléments traces métalliques(Cadmium, Plomb, Cuivre, Zinc et Nickel) des sols afin d'évaluer l'état environnemental et de préserver les potentialités et l'innocuité des sites de la région de Casablanca .

Un suivi des variations des paramètres physico-chimiques du sol $(\mathrm{pH}$, carbonates de calcium et matières organiques) est réalisé, suite à la dégradation de l'eau d'irrigation par l'utilisation d'eaux usées, et de l'impact de la pollution chimique. L'étude statistique par le test d'Anova montre des différences significatives concernant les paramètres physico-chimiques entre tous les sites. L'analyse des éléments traces métalliques montre que les sols des sites irrigués par Oued Hassar présentent des teneurs en Cadmium de 2,91 ppm et en Plomb de 137,61 ppm qui dépassent les normes préconisées par l'Organisation Mondiale de la Santé (O.M.S.), respectivement de 2 ppm et 100 ppm. Les teneurs en Cuivre dans les sols des sites irrigués par l'Oued Merzeg restent les plus élevées et sont de 896,62 ppm. Les teneurs en Zinc dans les sols proches de la cimenterie de Bouskoura sont prédominantes par rapport aux autres éléments et sont de 537,22 ppm. Dans les sols de MohammediaBenslimane, les teneurs de Cadmium sont de $2,36 \mathrm{ppm}$. Les teneurs en Plomb dépassent rarement 100 ppm. 
L'étude statistique par le test d'Anova montre des différences significatives entre tous les sites pour tous les éléments traces métalliques sauf pour le Nickel.

Mots clés : Qualité Environnementale, Qualité Agricole, Sol, Éléments Traces Métalliques, Région De Casablanca

\title{
Environnemental Status Of Soils In The Casablanca Region
}

\section{Benazzouz, \\ M. Talbi,}

Laboratory of Analytical chemistry and physical chemistry of materials Faculty of Sciences : Ben m'sick University Hassan II, Casablanca, Morocco

N. Saber,

High School of Technology Sidi Bennour, Morocco

Laboratory of devlopement and research

\begin{abstract}
Chemical pollution in the peri-urban area of Casablanca impacts the quality of agricultural soils.Five sites from the region of Casablanca have been studied: Oued Hassar, Oued Merzeg, Bouskoura, Benslimane-Mohammedia, and Bouznika.The objective is the comparative analysis of five trace metal elements in soils in order to assess the environmental state and to preserve the potential and harmlessness of sites in the Casablanca region.The environmental state of these sites requires knowledge of the physico-chemical parameters of the soil ( $\mathrm{PH}$, Calcium carbonates, organic matter), following the degradation of irrigation water by the use of wastewater or the impact of chemical pollution. The environmental state studied through metallic trace elements shows that the soils of sites irrigated by Oued Hassar have cadmium and lead contents which exceed the standards recommended by the World health organisation (WHO), respectively $2 \mathrm{ppm}$ and $100 \mathrm{ppm}$. The contents are respectively $2,91 \mathrm{ppm}$ and $137,61 \mathrm{ppm}$. The copper contents in the soils irrigated by the Merzeg wadi remain the highest.The contents are 896,62 ppm. The zinc contents in soils near the Bouskoura cement plant are predominant compared to the other elements.The contents are 537,22 ppm. In
\end{abstract}


Mohammedia-Benslimane soils, the cadmium contents are very variable, moderate and can exceed $2 \mathrm{ppm}$. Lead levels rarely exceed $100 \mathrm{ppm}$. The statistical study by the Anova test shows significant differences in the contents of metallic trace elements except for nickel.

Keywords : Environmental Condition, Agricultural Quality, Soils, Metallic Trace Elements, Casablanca Region

\section{Introduction}

La région de Casablanca est située au Centre-Ouest du Maroc. Elle est délimitée par l'Océan Atlantique à l'Ouest, la région de la Chaouia Ouardigha au Nord à l'Est et au Sud par la région de Settat et la province de Benslimane au Nord. Sa population représente $20,30 \%$ de la population totale du Maroc (Haut Commissariat au Plan) (HCP, 2010).

La région de Casablanca connait une forte urbanisation (Centre régional d'investissement, 2018). Le réseau routier couvre $5693 \mathrm{Km}$. L'équipement portuaire de Casablanca et Mohammedia ville avoisinante regroupe $55 \%$ du trafic routier national (Direction générale des collectivités locales, 2015)

L'Agriculture absorbe $53 \%$ de la population active de la région et représente $13 \%$ de la surface agricole utile du Maroc. C'est également une région fortement industrialisée, regroupant les industries de transformation, notamment les industries agro-alimentaires, chimiques (HCP, 2010)

Le produit intérieur brut (PIB) (2016) de la région a été estimé à $32 \%$ du PIB national (Direction générale de la région de Casablanca-Settat, 2015). C'est la première région industrielle et une grande agglomération qui connait des problèmes de détérioration de la qualité de l'air (Enajjari et al., 2016), ainsi que des risques de pollution par infiltration de la nappe phréatique par les eaux usées des rejets d'origines diverses. (Smahi et al., 2013).

A la périphérie et au Sud de Casablanca, la décharge de Médiouna accumule les déchets non traités et constitue une menace majeure de pollution (Iounes et al., 2018)

Oued Merzeg et Oued Hassar connaissent une grande détérioration de la qualité de l'eau car ils sont le réceptacle des eaux usées. Il y a une forte minéralisation et des perturbations des paramètres physico-chimiques de l'eau (Mounjid et al., 2014 ; Fouad et al., 2013) .Les eaux de ces Oueds sont utilisées en irrigation.

Les rejets hospitaliers liquides non traités menacent beaucoup plus l'environnement. Ils constituent une menace potentielle de transmissions de maladies et d'infections (Deblij et al., 2019). Le sol peut-être le réceptacle de contaminants divers. Les métaux lourds sont présents dans le sol à l'état de 
traces. Les activités industrielles, agricoles ou humaines contribuent à menacer l'équilibre des écosystèmes représentés par les nappes phréatiques, le sol, les plantes, les animaux et l'Homme (Noulos et al., 2018)

Les métaux lourds à l'état de traces ont un effet toxique sur différents types de sol. Ils perturbent les paramètres agronomiques. Il y a, en effet, des interactions entre les éléments traces métalliques dans le sol lorsqu'ils dépassent certaines concentrations et les paramètres agronomiques du sol comme la texture, le $\mathrm{pH}$, et les matières organiques (Adriano, 1986)

L'objectif de cette recherche est l'évaluation de la qualité environnementale des sols de la région de Casablanca qui peut-être perturbée par l'usage des eaux usées dans l'irrigation, ou par les effets de la pollution industrielle. Elle se focalise sur l'analyse comparative des éléments traces métalliques dans le sol. Les éléments traces métalliques recherchés sont : le Cadmium, le Plomb, le Cuivre, le Zinc et le Nickel . Ce sont des indicateurs de la qualité environnementale.

\section{Materiels Et Methodes}

\section{$\left.1^{\circ}\right)$ Le cadre géologique}

Le site de Casablanca fait partie de la Meseta Côtière. Il présente une couverture néogène et quaternaire sub-horizontale qui couvre le socle paléozoïque métamorphisé. La carte géologique de Casablanca-Mohammedia indique un socle d'âge Cambrien composé de quartzite et schistes, recouvert par des terrains de quelques dizaines de mètres d'épaisseur d'âge Miocène à quaternaire ancien présentés par des marnes, des limons et des sables plus ou moins calcifiés (Michard,1976)

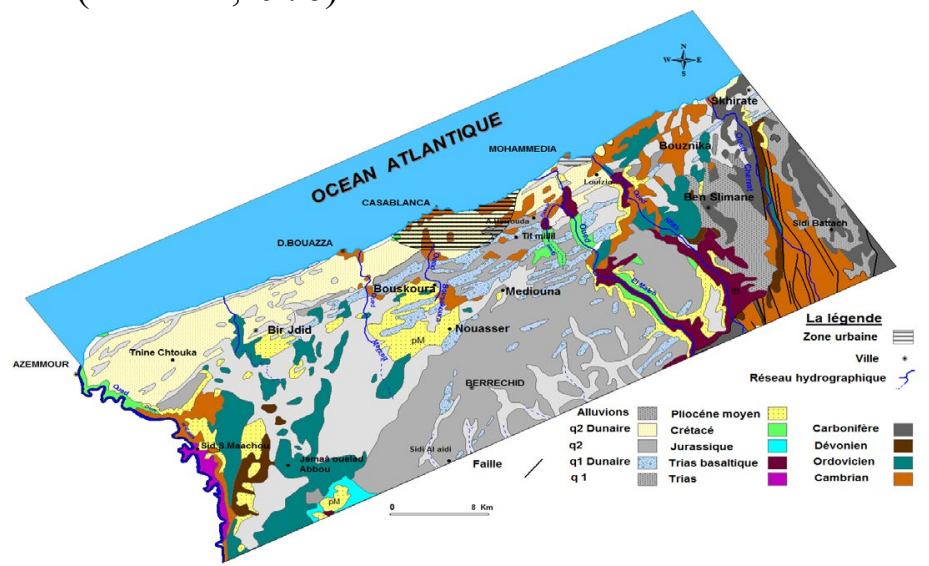

Figure1 : Cadre géologique de la zone d'étude

\section{$\left.2^{\circ}\right)$ Le climat}

Casablanca est sur la côte Atlantique marocaine. Elle se caractérise par un climat semi- aride avec des températures variant de $12,7^{\circ} \mathrm{C}$ en hiver et $21^{\circ} \mathrm{C}$ en été. Les cumuls pluviométriques varient entre 200 et $800 \mathrm{~mm}$ (Haut 
commissariat au plan, 2010) (Sebbar et al., 2012). En 2002, des précipitations importantes ont affecté le bassin d'Oued El Maleh et généré de nombreuses crues notamment à Mohammedia (Banque Mondiale, 2014)

\section{$\left.3^{\circ}\right)$ Zone d'étude}

Cinq sites sont choisis en fonction de l'usage des eaux usées dans l'irrigation, de la proximité de pollution industrielle ou d'usage excessif de fertilisants.

Le Sud-Est de Casablanca est irrigué par l'Oued Merzeg et, est réceptacle des eaux usées de la région de Berrechid.

Le Nord-Est de Casablanca est irriguée par l'Oued Hassar et est proche de la décharge de déchets de la région urbaine de Casablanca.

Le Nord de Casablanca à proximité de Mohammedia-Benslimane est représenté par des sols agricoles.

Le Nord de Mohammedia, à proximité de Bouznika, est représenté par des sols agricoles.

Le Sud de Casablanca est représenté par des sols agricoles à proximité de la cimenterie de Bouskoura.

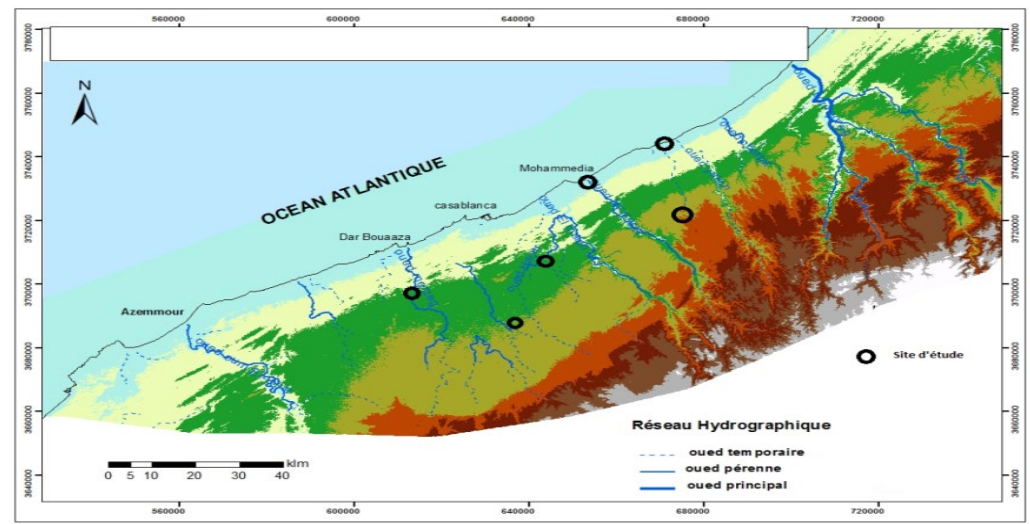

Figure 2 : Carte des sites étudiés

\section{$\left.4^{\circ}\right)$ L'échantillonnage}

Les échantillons au nombre de 163 , ont été prélevés au cours de la période de 2012-2015, dans le sol, dans les horizons de [0-20 cm], à la tarière manuelle au cours des périodes sèches. 300 à $500 \mathrm{~g}$ sont prélevés, séchés à l'air libre, broyés et tamisés par un tamis de $2 \mathrm{~mm}$ au laboratoire.

\section{Analyses physico- chimiques}

$5^{\circ}$ ) Analyse physique

La granulométrie a été déterminé à l'Institut Agronomique et Vétérinaire Hassan II à Rabat. 
La méthode utilisée sur les sols tamisés à $2 \mathrm{~mm}$ a été réalisée à la pipette de Robinson, en vue d'obtenir les pourcentages de chaque fraction granulométrique, l'argile est dans l'intervalle $(0-2 \mu \mathrm{m})$, les limons fins (2$20 \mu \mathrm{m})$, les limons grossiers $(20-50 \mu \mathrm{m}$, les sables fins $(50--200 \mu \mathrm{m})$, et les sables grossiers $(200-2000 \mu \mathrm{m})$.

$6^{\circ}$ ) Analyse Chimique

Le Carbone organique total est déterminé par la méthode de Walkley et Black. Les Carbonates de calcium sont dosés par la méthode de Bernard (Chamley,1966)

L'Acidité du sol $(\mathrm{pH})$ est mesurée par pHmétrie suivant la méthode de Mac Lean

L'Analyse des éléments traces métalliques est réalisée par spectrophotométrie plasma à couplage inductif au Laboratoire National pour la recherche scientifique et technique à Rabat.

\section{Analyses statistiques}

$7^{\circ}$ ) L'Analyse statistique est réalisée avec les logiciels SPSS 16 et EXCEL 2007

\section{Resultats Et Discussion}

$\left.1^{\circ}\right)$ Analyse texturale et analyse des paramètres agronomiques

Tableau1 : Analyse structurale des sols de la région de Casablanca

\begin{tabular}{|c|c|c|c|}
\hline Texture & Argile \% & Limon \% & Sable \% \\
\hline $\begin{array}{c}\text { Mohammedia } \\
\text { Min }\end{array}$ & $11,10 \%$ & $19,3 \%$ & $49,4 \%$ \\
Max & $20,5 \%$ & $40,7 \%$ & $59,8 \%$ \\
\hline Bouznika & & & \\
Min & $9,8 \%$ & $23,2 \%$ & $21,6 \%$ \\
Max & $27,41 \%$ & $51,1 \%$ & $55,6 \%$ \\
\hline $\begin{array}{c}\text { Oued Hassar } \\
\text { Min }\end{array}$ & $12,1 \%$ & $22,4 \%$ & $18,3 \%$ \\
Max & $46,1 \%$ & $69,6 \%$ & $33,4 \%$ \\
\hline Oued Merzeg & & & \\
Min & $5,4 \%$ & $5 \%$ & $74,7 \%$ \\
Max & $15 \%$ & $10,2 \%$ & $88,5 \%$ \\
\hline Bouskoura & & & \\
Min & $6,3 \%$ & $19,40 \%$ & $57,30 \%$ \\
Max & $8,3 \%$ & $35 \%$ & $57,30 \%$ \\
\hline
\end{tabular}

L'Analyse texturale comparative des sols montrent que les teneurs en sable représentent plus de la moitié de la composition dans tous les sites, suivies des limons et argiles.

Il y a un enrichissement en argile dans les sols irrigués par Oued Hassar dont les teneurs varient de $12,1 \%$ à $46,1 \%$. 
Les sols de la région de Casablanca sont : limononeux à limoneux-sableux à Oued Merzeg, limoneux à Mohammedia-Benslimane, Bouznika limoneuxsableux à Bouskoura et limono -sableux à argileux à Oued Hassar.

Tableau 2 : Analyse des paramètres agronomiques des sols de la région de Casablanca

\begin{tabular}{|c|c|c|c|}
\hline $\begin{array}{c}\text { Moyenne } \\
\text { Géométrique }\end{array}$ & $\mathbf{p H}$ & M.O \% & CaCO3 \% \\
\hline $\begin{array}{c}\text { Mohammedia- } \\
\text { Benslimane }\end{array}$ & 6,05 & 0,82 & 6,04 \\
\hline Bouznika & 6,58 & 1,09 & 1,04 \\
\hline Oued Hassar & 7,67 & 2,88 & 4,08 \\
\hline Oued Merzeg & 7,53 & 2,23 & 1,34 \\
\hline Bouskoura & 7,99 & 2,21 & 4,02 \\
\hline
\end{tabular}

L'analyse chimique montre que les valeurs moyennes de $\mathrm{pH}$ à Mohammedia-Benslimane et Bouznika sont acides de valeurs inférieures à $\mathrm{PH}$ 7. Ils sont comparables aux sols de la plaine du Saïss au Maroc (Touhtouh, et al., 2014). Les $\mathrm{pH}$ de ces sols sont inférieurs aux $\mathrm{pH}$ des sols de la plaine de Doukkala (Mohcine et al., 2015), de Jorf esfar (Moustaghfer et al., 2015) ou de la Chaouia (Rafik et al., 2015).

Les $\mathrm{pH}$ des sols irrigués par l'Oued Merzeg et Oued Hassar augmentent et sont supérieurs au pH des sols de Mohammedia-BenslimaneBouznika. Ils sont légèrement alcalins.

Les sols proches de la cimenterie de Bouskoura ont un $\mathrm{pH}$ alcalin.

Le $\mathrm{pH}$ des sols avoisinants la cimenterie de Bouskoura est probablement influencé par les poussières émanant de la cimenterie (Chemsi et al., 2014)

L'analyse de la matière organique montre que les teneurs sont plus élevées dans les sols irrigués par l'Oued Merzeg et l'Oued Hassar. Elles sont plus faibles dans les sols de Benslimane-Mohammedia et Bouznika. Ces teneurs sont supérieures aux sols de Sidi Rahal dans la région de la Chaouia (Rafik et al., 2014)] ou de ceux de Souss Massa dans le Sud du Maroc (El Oumlouki et al., 2014)

Les teneurs en matières organiques des sols irrigués par Oued Merzeg et Oued Hassar sont supérieures aux teneurs des sols de Benslimane Mohammedia, Bouznika (Zaakour et al., 2014) et de Tadla (Moughli et al., 2005) du Moyen Atlas (Bouabid et al., 2005)] et de la région de Meknes (Yaakoubi, 2010)

La qualité de l'eau d'irrigation affecte le $\mathrm{pH}$, et la conductivité électrique par une forte minéralisation et peut affecter les paramètres physicochimiques du sol. (Fouad et al., 2013)

Les teneurs en carbonates de Calcium sont variables, ce qui peut-être en relation avec la nature pédologique des sites de cette région. Elles sont 
inférieures aux teneurs trouvées dans la plaine du Saïss ou celles des sols au Moyen-Atlas (Touhtouh et al., 2014)

L'Analyse statistique par le test d'Anova montre qu'il y a des différences significatives concernant tous les sites étudiés concernant les paramètres physico- chimiques : $\mathrm{pH}$, matières organiques, carbonates de calcium $(\mathrm{p}<0,05)$.

Tableau 3 : résultats du test d'Anova

\begin{tabular}{|c|c|c|c|c|c|c|}
\hline & & $\begin{array}{c}\text { Somme } \\
\text { des } \\
\text { carrés }\end{array}$ & ddl & $\begin{array}{c}\text { Moye } \\
\text { nne } \\
\text { des } \\
\text { carrés }\end{array}$ & $\mathrm{F}$ & $\underset{n}{\text { Significatio }}$ \\
\hline \multirow{3}{*}{$\mathrm{PH}$} & Inter-groupes & 93,86 & 4 & 23,47 & 108,95 & 0,00 \\
\hline & Intra-groupes & 34,03 & 158 & 0,22 & & \\
\hline & Total & 127,89 & 162 & & & \\
\hline \multirow{3}{*}{ M.O. } & Inter-groupes & 105,78 & 4 & 26,44 & 67,70 & 0,00 \\
\hline & Intra-groupes & 61,72 & $\begin{array}{l}1 \\
5 \\
8 \\
\end{array}$ & 0,39 & & \\
\hline & Total & 167,49 & 162 & & & \\
\hline \multirow{3}{*}{$\mathrm{CaCO} 3$} & Inter-groupes & 948,66 & 3,00 & 316,22 & 29,94 & $\begin{array}{c}\ldots \ldots \ldots \ldots, 0 \\
0\end{array}$ \\
\hline & Intra-groupes & 1489,33 & 141,00 & 10,56 & & \\
\hline & Total & 2437,99 & 144,00 & & & \\
\hline
\end{tabular}

Cela montre des différences entre les sites qui dépendent des facteurs climatiques, du type d'irrigation, du type de cultures et d'interactions des sols avec les facteurs environnementaux.

Ces facteurs influent sur la fertilité des sols. Parmi les recommandations proposées pour préserver le maintien du niveau de fertilité des sols, il y a l'apport adéquat de fumure minérale et organique, et le parcage des bovins (Adifou et al., 2019). La gestion de la fertilité au cours des pratiques agricoles traditionnelles utilise aussi la rotation culturale ou la jachère de longue durée. L'introduction de méthodes récentes nécessite l'usage d'engrais minéraux ou organiques (Akpo et al., 2016). L'utilisation de légumineuses précédant d'autres cultures est mentionnée pour maintenir le maintien du niveau de fertilité des sols .Les légumineuses grâce à leur potentiel fixateur d'azote ct l'enrichissement du sol en azote par la décomposition de leurs racines.

Elles contribuent ainsi à la dépollution azotée, limitent l'épandage par les engrais chimiques et favorise la restauration de sols pauvres (Lahdachi et al., 2015).

L'état environnemental des sols des cinq sites étudiés est révélé à travers l'analyse des éléments traces métalliques comme suit :

$2^{\circ}$ ) Eléments Traces Métalliques dans les sols de la région de Casablanca 


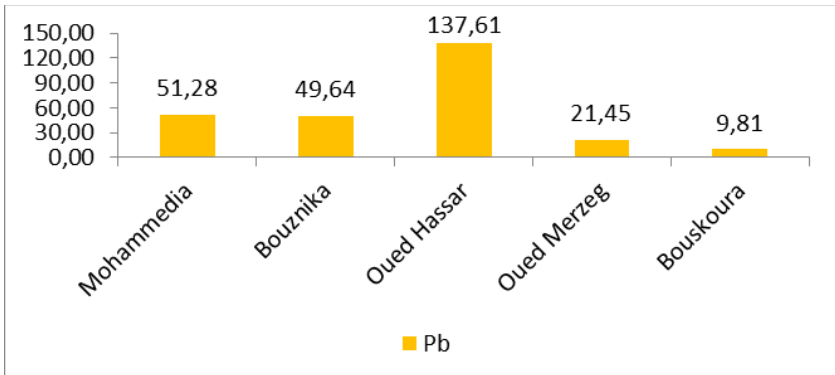

Figure 3 : Teneurs en ppm du Plomb dans les sols de sites de la région de Casablanca.

Les teneurs en plomb des sols irrigués par Oued Hassar sont de 137,61 ppm,de 51,28 ppm dans les sols des parcelles agricoles de Mohammedia ,de 49,64 ppm dans les sols agricoles de Bouznika,de 21,45 ppm dans les sols irrigués par Oued Merzeg et de 9,81 ppm dans les sols avoisinants la cimenterie de Bouskoura.Le seuil critque tolérable dans le sol établi par l'O.MS. est pour le Plomb de 100 ppm.

Il est dépassé au niveau des sols irrigués par Oued Hassar.Les teneurs en plomb des sols de la région de Casablanca sont supérieures aux teneurs dans les sols de Marrackech, Rabat-Salé, Kénitra et Settat (Kennou et al., 2014 ; El Idrissi, 2009 ; Kao et al., 2007)

L'analyse des teneurs en Cadmiun révèle des teneurs supérieures à 2 ppm seuil tolérable par l'O.M.S dans les sols de Bouskoura (5,07 ppm), dans les sols irrigués par Oued Hassar (2,91 ppm), et dans les sols de MohammediaBenslimane (2,31 ppm).

Les teneurs en Cadmium dans les sols de ces sites suscitent des questionnements sur l'origine de cette contamination, et de l'impact des facteurs physico-chimiques de ces sols.

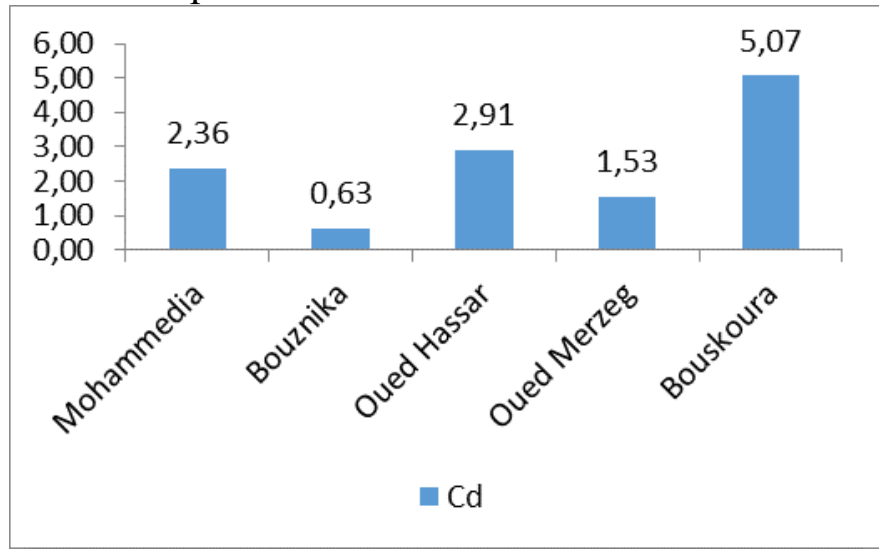

Figure 4 : Teneurs en ppm du Cadmium dans les sols de la région de Casablanca

Les sols irrigués par Oued Merzeg et les sols de Bouznika présentent des teneurs inférieures à $2 \mathrm{ppm}$. 
Concernant les contaminants majeurs, le Cuivre enregistre des teneurs très élevées de 896,62 ppm et supérieures au seuil indiqué par l'O.M.S (100ppm) dans les sols irrigués par Oued Merzeg. (figure 5)

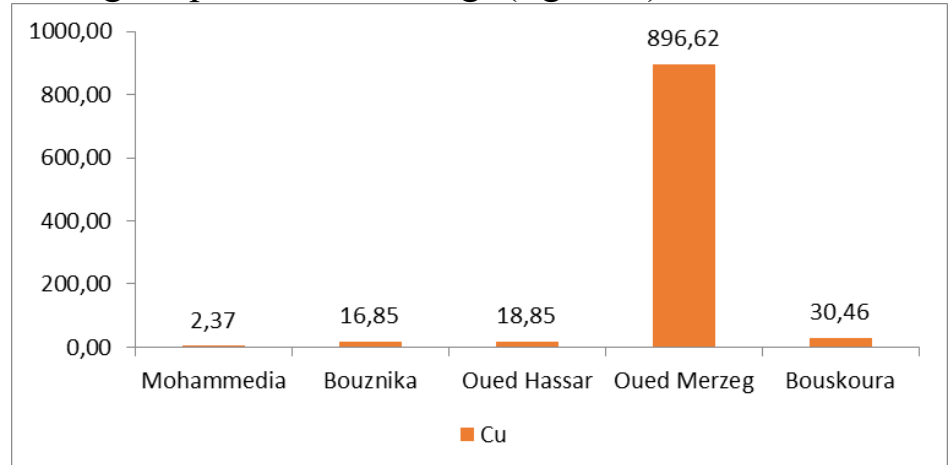

Figure 5 : Teneurs en ppm du Cuivre dans les sols des sites de la région Casablanca

Les teneurs en Zinc sont prédominantes dans les sols avoisinants la cimenterie de Bouskoura (537,22 ppm) et dans les sols de Bouznika (145,55 ppm).Elles sont supérieures au seuil tolérable par l'O.M.S.de $100 \mathrm{ppm}$. (figure 6)

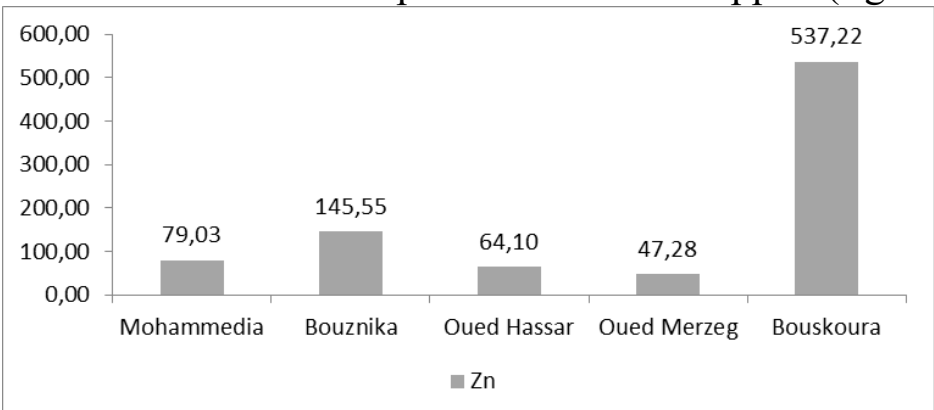

Figure 6 : Teneurs en ppm du Zinc dans les sols des sites de la région de Casablanca

Les teneurs en Nickel dans les sols de la région de Casablanca sont modérées. Il ya peu de variations observées entre les différents sites. Elles restent inférieures au seuil tolérable par l'O.M.S. de 50 ppm.(figure 7)

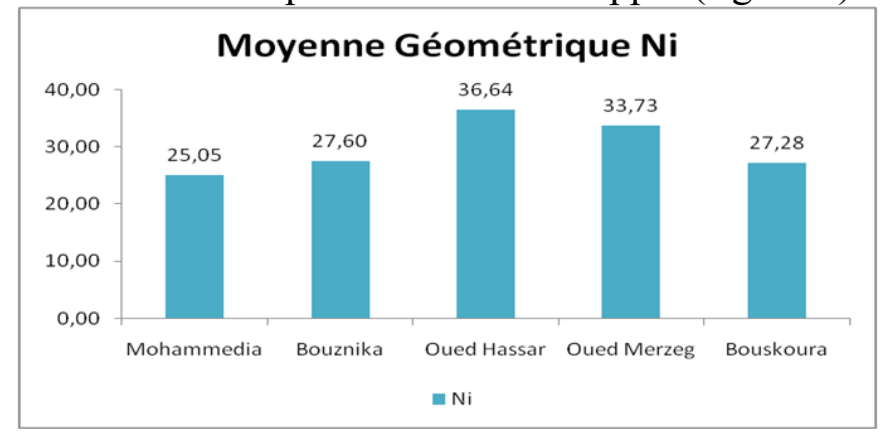

Figure 7 : Teneurs en ppm du Nickel dans les sols des sites de la région de Casablanca 
Afin de compléter l'analyse des éléments traces métalliques dans chaque site, une analyse comparative entre les sites est réalisée et soutenue par le test statistique d'Anova. Ce test permet de révéler des différences significatives lorsque $\mathrm{p}<0,05$.

Tableau 3 : résultats du test statistique

\begin{tabular}{|c|c|c|c|c|c|c|}
\hline & & $\begin{array}{c}\text { Somme des } \\
\text { carrés }\end{array}$ & ddl & $\begin{array}{l}\text { Moyenne } \\
\text { des carrés }\end{array}$ & $\mathrm{F}$ & $\begin{array}{c}\text { Significatio } \\
\mathrm{n}\end{array}$ \\
\hline \multirow{3}{*}{$\mathrm{Cd}$} & Inter-groupes & 491,67 & 4 & 122,92 & 21,47 & 0,00 \\
\hline & Intra-groupes & 904,72 & 158 & 5,73 & & \\
\hline & Total & 1396,39 & 162 & & & \\
\hline \multirow{3}{*}{$\mathrm{Cu}$} & Inter-groupes & 14151644,90 & 4 & 3537911,23 & 216,41 & 0,00 \\
\hline & Intra-groupes & 2583072,27 & 158 & 16348,56 & & \\
\hline & Total & 16734717,18 & 162 & & & \\
\hline \multirow{3}{*}{$\mathrm{Ni}$} & Inter-groupes & 4593,29 & 4 & 1148,32 & 1,78 & 0,14 \\
\hline & Intra-groupes & 102112,27 & 158 & 646,28 & & \\
\hline & Total & 106705,56 & 162 & & & \\
\hline \multirow{3}{*}{$\mathrm{Pb}$} & Inter-groupes & 274304,97 & 4 & 68576,24 & 118,85 & 0,00 \\
\hline & Intra-groupes & 91169,21 & 158 & 577,02 & & \\
\hline & Total & 365474,19 & 162 & & & \\
\hline \multirow{3}{*}{$\mathrm{Zn}$} & Inter-groupes & 19807073,57 & 4 & 4951768,39 & 33,57 & 0,00 \\
\hline & Intra-groupes & 23306480,63 & 158 & 147509,37 & & \\
\hline & Total & 43113554,20 & 162 & & & \\
\hline
\end{tabular}

Il y a des différences significatives des teneurs en éléments traces métalliques dans les sols entre tous les sites de la région de Casablanca excepté pour le Nickel. Cela signifie que le degré de contamination ainsi son origine sont différents. Ces différences sont confirmées par les résultats du calcul de l'Indice de pollution.

Le calcul de l'indice de pollution indique une valeur supérieure à 1 dans les sols irrigués par l'Oued Merzeg. Le degré de pollution est plus important dans les sols de l'Oued Merzeg ou l'Oued Hassar. Les teneurs de Cadmium suivent l'ordre décroissant de Bouskoura, Oued Hassar, Mohammedia-Benslimane, Oued Merzeg et Bouznika. Les teneurs de plomb augmentent dans l'ordre de Bouskoura, Oued Merzeg, Bouznika, Mohammedia-Benslimane et Oued Hassar.Les teneurs en Cuivre suivent un ordre croissant dans l'ordre de Mohammedia-Benslimane, Bouznika, Oued Hassar, Bouskoura et Oued Merzeg. Les teneurs en Zinc augmentent dans l'ordre Oued Merzeg, Oued Hassar, Mohammedia-Benslimane, Bouznika et Bouskoura. Les teneurs en Nickel varient suivant un ordre croissant de 
Mohammedia-Benslimane, Bouznika, Bouskoura, Oued Merzeg, et Oued Hassar.

Le calcul de l'Indice de Pollution révèle une différence spatiale avec une diminution dans le sens des sites Oued Merzeg, Oued Hassar, Mohammadia-Benslimane, Bouskoura et Bouznika.

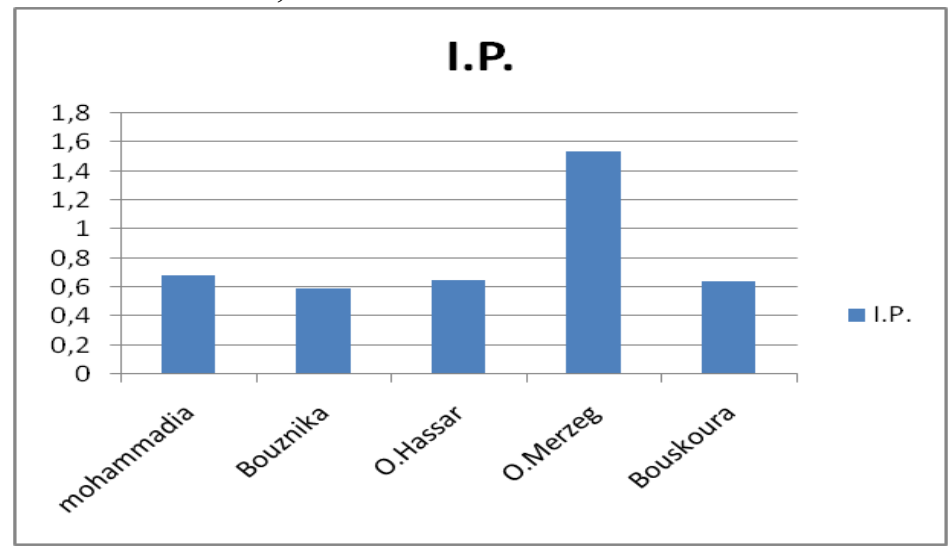

Figure 8 : Variation de l'Indice de Pollution dans la région de Casablanca

Des études préliminaires ont montré des variations entre les parcelles agricoles, suivant la proximité d'un réseau routier important (Zaakour et al., 2014) ou la proximité d'une source de pollution chimique (Chemsi et al., 2014) ou encore l'impact de l'irrigation des sols par les eaux usées (Matech et al., ; Siba et al., 2018).Ces mêmes variations ont été observées par une étude réalisée en Europe, impliquant les mêmes sources de pollution d'origine anthropique (Noulos, 2018).Les contaminants les plus souvent identifiés dans les sols en Europe et dans les nappes phréatiques de sites contaminés en Europe sont les métaux lourds. En France, ce sont les anciennes régions minières et régions les plus urbanisées qui concentrent le plus de sites et sols pollués. L'île de France, Nord Pas de Calais et Rhône Alpes comptabilisent à elles seules $40 \%$ des sites et sols pollués. Ce sont les sols situés en Europe de l'Ouest et le pourtour Méditerranéen qui présentent des teneurs en éléments traces métalliques élevées (Noulos et al, 2018)

L'origine des contaminations anthropiques peut être liée, à l'usage excessif de fertilisants phosphatés, l'infiltration des nappes phréatiques et sols par les lixiviats de compostage des déchets urbains. Dans ce cas, c'est le Cadmium qui prédomine comme éléments majeur (Chu et al, 2019). La proximité de trafic routier est source de pollution surtout par le Plomb et le Cadmium (Akbar et al, 2006)

L'irrigation des sols par les eaux usées est un facteur de contamination surtout par le Plomb, Cuivre, et Zinc. (Kumar et al, 2018). Dans l'ordre les risques écologiques dans les sols sont majeurs dans le sens décroissant : $\mathrm{Cd}$, $\mathrm{Fe}, \mathrm{Mn}, \mathrm{Cr}, \mathrm{Cu}, \mathrm{Ni}, \mathrm{Pb}, \mathrm{As}, \mathrm{Co}$. 
L'analyse statistique par le test d'Anova montre une différence statistique entre tous les éléments traces métalliques de tous les sites de la zone étudiée sauf pour le Nickel, ce qui confirme les variations spatiales supposées liées aux sources de contaminations ainsi qu'à l'impact des paramètres physicochimiques et structuraux du sol.

Les métaux sont très toxiques et rémanents (Xiang et al., 2016). Les végétaux les absorbent par voie racinaires et aériennes. Ils se retrouvent dans les cultures qui sont consommées par l'Homme (Wu et al., 2015) Les métaux lourds sont absorbés ou inhalés par contamination aigue ou chronique et entrainer ainsi des problèmes de santé graves (Chang et al., 2014) Le Plomb, le Cadmium, le Cuivre, l'Arsenic sont des éléments traces métalliques couramment retrouvés dans les écosystèmes. Les métaux lourds peuvent causer plusieurs effets délétères chez l'Homme et affecter les organes vitaux (Tang et al, 2003). Ils ont un effet pernicieux sur la fertilité parce qu'ils sont des perturbateurs endocriniens (Eum et al., 2014).

Pour limiter les effets négatifs des contaminants chimiques, la phytoremédiation et l'usage d'extraits naturels de plantes comme biopesticides aboutissent à préserver l'inocuité des sols, des chaines trophiques, la santé humaine et l'environnement global. (Sana et al., 2019; Bachiri et al., 2017).

\section{Conclusion}

Les sols de la région de Casablanca sont limoneux à MohammediaBenslimane-Bouznika, limoneux à limoneux- sableux à Oued Merzeg, limoneux-sableux à Bouskoura, limoneux -sableux à argileux à Oued Hassar . Les $\mathrm{pH}$ varient de 6,05 dans les sites de Mohammedia-Benslimane-Bouznika, à 7,99 dans les sites de Bouskoura. Les carbonates de calcium sont plus faibles à Oued Merzeg suivant l'ordre décroissant : Mohammedia-Benslimane-Oued Hassar-Bouskoura-Oued Merzeg.

Les teneurs traces métalliques montrent des teneurs en plomb les plus élevées et dans l'ordre suivant: Oued Hassar Mohammedia-BenslimaneBouznika- Oued Merzeg-Bouskoura.Les teneurs en Cuivre dans le site d'Oued Merzeg restent les plus élevés .par contre les teneurs en Zinc sont prédominantes dans les sols de Bouskoura.

L'étude statistique par test Anova montre des différences significatives entre les sites Nord, Nord-Est, Sud de Casablanca, et Nord de Mohammedia concernant les teneurs traces métalliques $(\mathrm{Cd}, \mathrm{Pb}, \mathrm{Cu}, \mathrm{Zn})$ sauf pour le $\operatorname{nickel}(\mathrm{Ni})$.

Ces différences révèlent que l'état environnemental des sols de la zone urbaine et périurbaine de Casablanca nécessite un suivi strict afin de préserver une qualité agricole acceptable, car elle est tributaire de la qualité des eaux d'irrigation .et de la pollution chimique. Un contrôle régulier du traitement des 
eaux usées ainsi que des déchets industriels et des fertilisants agricoles est à envisager.

\section{Remerciements}

Mes remerciements sont adressés aux personnes ayant contribué à élaborer et à améliorer la qualité de ce manuscrit.

\section{References:}

1. Adifou, F.S., Yabi, I.,Balogoun, I.,Dossou J., Saïdou, A., (2019). Caractérisation socio-économique des systèmes de culture à base d'Igname dans trois zones agro-écologiques pour une gestion durable au Bénin.European Scientific Journal, 15, (12), pp214-232.

2. Adriano, D.C., (1986)

3. Traces elements in the terrestrial environnement. Spinger-Verlag, New-York.

4. Afnor NF-31-107, 2003

5. Qualité du sol : Détermination de la distribution granulométrique des particules du sol (méthode à la pipette de Robinson).

6. Akbar, K., Hale, M., Headley, A., Athar, A., (2006) Heavy metal contamination of roadsides soils of Northern England. Soil and Water Res., 1, (4) pp158-163.

7. Akpo, M.A.,Saidou, A.,Balogoun, I.,Yabi, I.,BioBigou, I.B., (2016) Evaluation de la performance des pratiques de la gestion de la fertilité des sols dans le bassin de la rivière Okpara au Bénin.European Scientific Journal, 12, (33), pp370-390.

8. Bachiri, L., Bamou, M.,Echchegada,G.,Ibijbijen,J., J.,El Rhafari,L., Nassiri, L.,(2017) Composition chimique et activité antimicrobienne des huiles essentielles de deux espèces de lavande: Lavandula Dendata Spp.et Lavandulla Peduncultata Spp.Peddunculata European Scientific Journal, 13,(21), pp293-311.

9. Banque mondiale, ( 2014) Adaptation aux changements climatiques et aux désastres naturels des villes côtières d'Afrique du Nord. Evaluation des risques en situation actuelle et à l'horizon2030, Egis Becom International/AUDF/BRGM. Maroc. 120p.

10. Bouabid, A., et Moustaoui, D., (2005) Fertilisation en arboculture fruitière dans le Saïss et le Moyen Atlas.Processing du colloque : Gestion environnementale de l'Agriculture, pp153-164.

11. Chamley,1966 Guide des techniques de laboratoire de géologie marine de Luminy, France, 198p.

12. Chang, S.H.,Cheng, B.H., Lee, S.,L., (2014) Low blood lead concentration in association with infertility in women. Environ. Res., 101, pp380-386. 
13. Centre régional d'investissement, (2018) Données socio-économiques de Casablanca-Settat 86p.

14. Chemsi, Z.,,Saber,

N.,Zaakour,F.,Matech, F.,Z.,Marrackchi,C ;,Saber,N., (2015) Distribution des éléments traces métalliques dans les sols avoisinants la cimenterie de Bouskoura. European Scientific journal, 10, (27), pp207-223.

15. Chu, Z.,Fan, X.,Wang,W.,Huang W., (2019) Quantitative evaluation of heavy metals pollution hazards and estimation of heavy metals environmental coasts in leachate during food waiste composting.Waiste Management, 84, pp119-128.

16. Deblij, S.,Bahlaouane, B.,Boutaleb.,N.,El Antri.,S., (2019) Diagnostic de la gestion des affluents liquides hospitaliers de la région de Casablanca-Settat. European Scientific Journal, 15 , 6, pp171-190.

17. Direction générale des collectivités locales, (2015) Monographie générale de la région de Casablanca-Settat. Maroc.52p.

18. Direction générale de la région de Casablanca-Settat, (2018) Monographie de la région de Casablanca-Settat. Maroc.19p.

19. El Idrissi , M., (2009) Caractérisation de quelques sites de pollution des sols périurbains par les traces métalliques. Rabat-Maroc. Mémoire de troisième cycle pour l'obtention du diplôme d'ingénieur Agronome. Institut Agronomique et vététinaire-HassanII.

20. El Oumlouki.,K.,Moussadek.,L.,Zouahri.,Dakak.,Z,Chati.,M.,El Amrani, (2014) Etude de la qualité physico-chimique des eaux et des sols de la région de Massa. Maroc. J.Mater.Environ.Sc., 5, pp164-171.

21. Ennajari, M., Meccharka-Tahiri S.Tessier, J.F., (2000) La Pollution atmosphérique. Qu'en est-il au Maroc ? Pollution atmosphérique, 165, pp91-96.

22. Eum, K.D., Weisskopf, M.G., Nie, L.H., (2014) Cumulative lead exposure and age at menopause in the nurse health study cohort. Environ. Health Perspect., pp 122-129.

23. Fouad, S., Cohen, N., Hajjami, K., Chlaida, M., (2014) Qualité physico-chimique et contamination de l'Oued Hassar: impact des eaux usées de la localité de Médiouna (périurbain de Casablanca).Revue internationale des Sciences et technologie, 10, (1) pp91-102.

24. Haut commissariat au plan, (2010) Monographie de la région du grand Casablanca, Maroc, 152p.

25. Iounes, N.,Sassioui I., S.,Ait Ouadi, A., Bassou M., Namoussi S., Merbouh Ch., Mestaghnmi H., El Amrani, S ., (2018) Reabilitation of Médiouna wildlandfill caracterisation of biogaz and treatment of landfill leachgate. Layris Journal, 35, pp107-117 
26. Kao T.,Mejahed K.,Bouzidi A., (2007) Evaluation de la pollution metallique dans les sols agricoles irrigués par les eaux uées de la ville de Settat-Maroc. Bulletin de l'Institut scientifique,Rabat,Section Sciences de la vie, 29, 89-92.

27. Kennou, B., El Meray, M., Romane, A., Bodjana, B., Arjouni Y., (2014) Assesment of heavy metal availability (Pb,Cu,Cr,Cd, $\mathrm{Zn}$ ) and speciation in contaminated soils and sediments. proceedings E3D.

28. Kumar, M., Sharma A., Kaur, P., Singh, G., Bali, A., Bhardway, R., Thurkrai, A.,Lerda A., (2018), Pollution assesment of heavy metals in India and ecological risk assesment ;Astate of art. Grounwater for sustainable Development, 8 , pp122-123.

29. Lahdachi, F.Z. Nassiri, L., Ibjibijen, J., Mokhtari., F., (2015) Aperçu sur les acacias spontanés et introduits au Maroc . European Scientific Journal, 11, (23), 88-101.

30. Mac Lean E.O., 1982 Methods of soil analysis. Second Ed .Agronomy, 9 , Soil Society of America, pp199-244

31. Matech, M., Zaakour, F., Chemsi, Z., Benazzouz, I., Saber, N., (2014) Concentrations en éléments traces métaliques dans les sols irrigués par les eaux usées versées dans l'Oued Merzeg(Casablanca-Maroc) . European Scientific Journal, 10, 29, pp121-138.

32. Mohcine, H., Saber, N., Moustarhfer, K., Matech, .Z., Marrackechi C., 2015 Qualité agronomique des terrains agricoles de l'Oulja-Sahel Doukkala-Maroc. European Scientific Journal, 11, (21), pp168-181.

33. Moustarhfer, K., Saber, N., Mohcine, H., Rafik, F., Zaakour, F., Matech , F.Z.,(2015) Qualité agronomique des sols sous cultures maraichères de la région de Jorf Lasfar (Doukkala-Maroc). European Scientific Journal, 11, (24) pp 54-69.

34. Moughli , L., et Cherkaoui, F.Z., (2005) Fertilisation minérale rationnelle des cultures et élaboration des fiches éco-conseils, cas de la betterave à sucre. Gestion environnementale de l'agriculture. ActesEditions, pp141-152.

35. Noulos, C., Tziouvalekas, M.,Karyotis , T., (2018) Zinc in soils,water and food crops. Journal of trace elements in Medecine and Biology, 2, pp252-260.

36. Rafik, F., Mohcine, H., Moustaghfir, K., Zaakour, F., Matech, F.Z., (2015) Qualité agronomique des sols sous cultures maraichères de la régionde Sidi Rahal (Chaouia côtière) . European Scientific Journal, 11, (27) pp48-63.

37. Sana, A., Quassim, A., Zahir, A., Sobia A., Hafiz, A.Z., (2019) Phytoremediation :Environmental sustainable way for reclamation of heavy metal polluted soils . Ecology and Environmental Safety,174, pp714-727. 
38. Sebbar, A., Fougrach H., Hsain M., Badri M., 2012. Actes du XXVIième colloque de l'association nationale de climatologie de Grenoble, France. pp709-711.

39. Smahi, D., Hammoumi, O.E., Fekri, A., (2013) Assessment of the impact of landfill on groundwater quality a case study of the Médiouna site. Journal water ressources and protection, 5, pp440-445.

40. Siba, A., El Jaafari, S.,Mokhtari, F.,(2018) Pollution bactérienne et toxique des eaux de rejets industriels et domestiques du littoral atlantique (Casablanca-Est-Maroc). European scientific Journal , 14, (12), pp425-431.

41. Touhtouh, D., Moujahid, Y., El Faleh, E.M.,El Halimi, R., (2014) Caractéristiques physico-chimiques de trois types de sols du SaïssMaroc. J. Mater.Environ.sci.,5,(3), pp 1524-1534.

42. Tang, S., et Zhu, Z.Q., (2003) Adverse reproduction effects in female workers of lead battery Int.J.Occup.Med.Environ. Health, 16, pp359361.

43. Yaakoubi, A., (2010) Biotransformations des margines par voie biologique et impact de leur épandage sur la qualité du sol : cas de la vigne dans la région de Meknes. Faculté des Sciences de Meknes. Maroc. Thèse de Doctorat-Es -Sciences

44. Walkey, A., et Black I.A ., (1934) Examination of the detjareff method for determination soil organic matter and proposed modification of chromic acid titration method. Soil Science. 62, pp 251-254.

45. Wu, S., et Peng Zhang X., (2015) Levels and health risk assessments of heavy metals in urbans soils in Donggan, China . Environ, Geochem. Health, 32, pp 1-19 